\author{
Michele Feleppa \\ Fred D. Sheftell \\ Luciana Ciannella \\ Amedeo D'Alessio \\ Giancarlo Apice \\ Nino N. Capobianco \\ Donato M.T. Saracino \\ Walter Di Iorio \\ Marcelo E. Bigal
}

\section{A new questionnaire for assessment of adverse events associated with triptans: methods of assessment influence the results. Preliminary results}

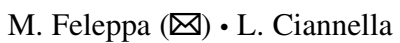

A. D'Alessio • G. Apice • N.N. Capobianco

W. Di Iorio

Department of Neuroscience,

Hospital "G. Rummo",

Via dell' Angelo, I-82100 Benevento, Italy e-mail: michele.feleppa@ao-rummo.it

Tel.: +39-0824-57492/57465

Fax: +39-0824-57465

F.D. Sheftell

Department of Psychiatry,

New York Medical College, NY, USA

F.D. Sheftell • M.E. Bigal

The New England Center for Headache,

Stamford, CT, USA

\section{D.M.T. Saracino}

Department of Medical Up-Grading, Hospital "G. Rummo", Benevento, Italy

M.E. Bigal

Department of Neurology,

Albert Einstein College of Medicine,

NY, USA

\begin{abstract}
Triptans are the treatment of choice for migraine sufferers with disabling attacks. However, the proportion of patients reporting side effects after any acute treatment may vary in regard to the method of assessment. This study was conducted in a neurology office focusing on headache in Italy. We prospectively surveyed adult headache sufferers who had been using the same triptan for at least 3 months (from March 2001 to May 2003). Participants were asked about their headache and treatment history. Subjects then completed a standardized questionnaire, assessing adverse events in two different ways. First, subjects were asked if they had any adverse events when using the triptan. If they answered yes, they were asked to list them and grade their severity as mild, moderate, or severe. After returning the first part of the questionnaire, subjects received a second form, where 49 possible adverse events were listed. Most of them were known triptan side effects; some confounders (side effects not expected to be related with triptan use) were added. We contrasted and correlated both sets of answers. We surveyed 108 subjects, $(87.1 \%$ female, mean age 39.5 years). Most
\end{abstract}

patients $(65.5 \%)$ reported no side effects in the unprompted questionnaire. However, most of them $(54.1 \%)$ reported at least one side effect in the prompted questionnaire. The majority of patients that reported side effects in the unprompted questionnaire said they had only one adverse event, while most reported two or more side effects in the prompted questionnaire. Both in the unprompted and in the prompted questionnaires, most side effects were rated as mild or moderate. Two (1.9\%) subjects graded their adverse events as severe in the prompted questionnaire, but had not self-reported them. We conclude that when assessing the adverse events of triptans (or any class of medication), the method of data collection may dramatically influence the results.

Key words Triptans $\cdot$ Adverse events $\cdot$ Methods of assessment 


\section{Introduction}

Triptans are the treatment of choice for migraine sufferers with disabling attacks [1]. Six oral triptans, in different doses and formulations, are available in Italy [2, 3]. Tolerability is the second most important attribute of an acute treatment for naïve migraine sufferers [4]. Triptans have different tolerability profiles [5]. Sheftell et al. recently conducted a study showing that from $7 \%$ to $39 \%$ (depending on the triptan and the formulation) of migraine sufferers switching triptans did so because of side effects [6].

Major methodological issues involve the assessment of tolerability. Safety and tolerability have different definitions and different methods of assessment [7]. In addition, the proportion of patients reporting side effects after any acute treatment may vary in regard to the method of assessment. This issue is also of major significance regarding triptans. Because we consider that the methods of assessing tolerability may dramatically influence the results, in this study we prospectively contrasted two methods of assessing adverse events (prompted and unprompted) in headache sufferers using triptans.

\section{Patients and methods}

We prospectively surveyed adult headache sufferers from March 2001 to May 2003 fulfilling the following inclusion criteria:

1. Diagnosis of migraine made according to the first edition of the International Headache Society (1988) criteria [8], or diagnosis of transformed migraine without medication overuse according to the criteria proposed by Silberstein and Lipton [9]

2. Patients had been using the same triptan for at least 3 months.

3. Patients had been followed up at the center for at least 1 year At the time this study was performed, frovatriptan and naratriptan were not available.

Participants were first asked about their headache and treatment history. After being included, all subjects completed a standardized questionnaire, assessing adverse events in two different ways. First, subjects were asked if they had any adverse events when using the triptan (unprompted questionnaire) (Fig. 1). If they answered yes, they were asked to list them and grade their severity as mild, moderate, or severe and their time relief in minutes.

After returning the first part of the questionnaire, subjects received a second form. The second form was answered on the same day, but the subjects did not have the opportunity to check their answers in the first questionnaire. In the second questionnaire (prompted questionnaire), 49 possible adverse events were



Fig. 1 Questionnaire (simplified) for unprompted and prompted side effects 
listed (Table 1). Most of them were known triptan side effects, chosen from the PDR and from the pivotal clinical trials; some confounders (side effects not expected to be related with triptan use) were added.

The main objective of this study was to contrast and correlate both sets of answers (prompted and unprompted). Data were analyzed using Stata (Intercooled Stata 6.0 for Windows, College Station, TX). The chi-square test was used to determine statistical significance unless otherwise indicated. The number and severity of side effects in both methods were assessed using the chi-square test for trends.

\section{Results}

We surveyed 108 subjects $(87.1 \%$ female, mean age 39.5 years) (Table 1). The vast majority of the participants had migraine $(94.6 \%)$. Most patients were using rizatriptan $(39.8 \%)$, followed by almotriptan $(21.3 \%)$ (Table 2$)$. The majority of patients $(65.5 \%)$ reported no side effects in the unprompted questionnaire. However, most of them $(54.1 \%)$ reported at least one side effect in the prompted questionnaire (Fig. 2). Most patients who reported side effects in the unprompted questionnaire said they had only one adverse event, while most reported two or more side effects in the prompted questionnaire. Both in the unprompted and in the prompted questionnaires, most side effects were rated as mild or moderate (Table 3).
Table 1 Demographic characterization

$\mathrm{n}(\%)$

$\begin{array}{lrr}\text { Diagnosis } & & \\ \quad \text { Migraine } & 102 & (94.6) \\ \quad \text { Transformed migraine } & 6 & (5.4) \\ \text { Male } & 14 & (12.9) \\ \text { Female } & 94 & (87.1) \\ \text { Age, mean (SD) } & 39.5 & (12.2)\end{array}$

Table 2 Triptan being used when patients were enrolled in the study. Surveyed patients $n=108$

n $(\%)$

\begin{tabular}{lrr}
\hline Sumatritptan & 11 & $(10.2)$ \\
Tablet $100 \mathrm{mg}$ & 7 & $(4.7)$ \\
Tablet $50 \mathrm{mg}$ & 11 & $(7.4)$ \\
Nasal spray $20 \mathrm{mg}$ & 0 & \\
Injection $(6 \mathrm{mg})$ & 0 & \\
Zolmitriptan & 14 & $(12.9)$ \\
Tablet $5 \mathrm{mg}$ & 2 & $(1.4)$ \\
Tablet $2.5 \mathrm{mg}$ & 12 & $(8.1)$ \\
Dissolving tablet & 5 & $(3.4)$ \\
Rizatriptan & 43 & $(39.8)$ \\
Tablet $10 \mathrm{mg}$ & 26 & $(17.6)$ \\
Tablet $5 \mathrm{mg}$ & 1 & $(6.7)$ \\
Dissolving tablet & 30 & $(20.7)$ \\
Eletriptan & 17 & $(15.8)$ \\
Almotriptan & 23 & $(21.3)$ \\
\hline
\end{tabular}

Fig. 2 Proportion of subjects reporting side effects in the unprompted and prompted questionnaires. $* p<0.001 ; * * p<0.0001$

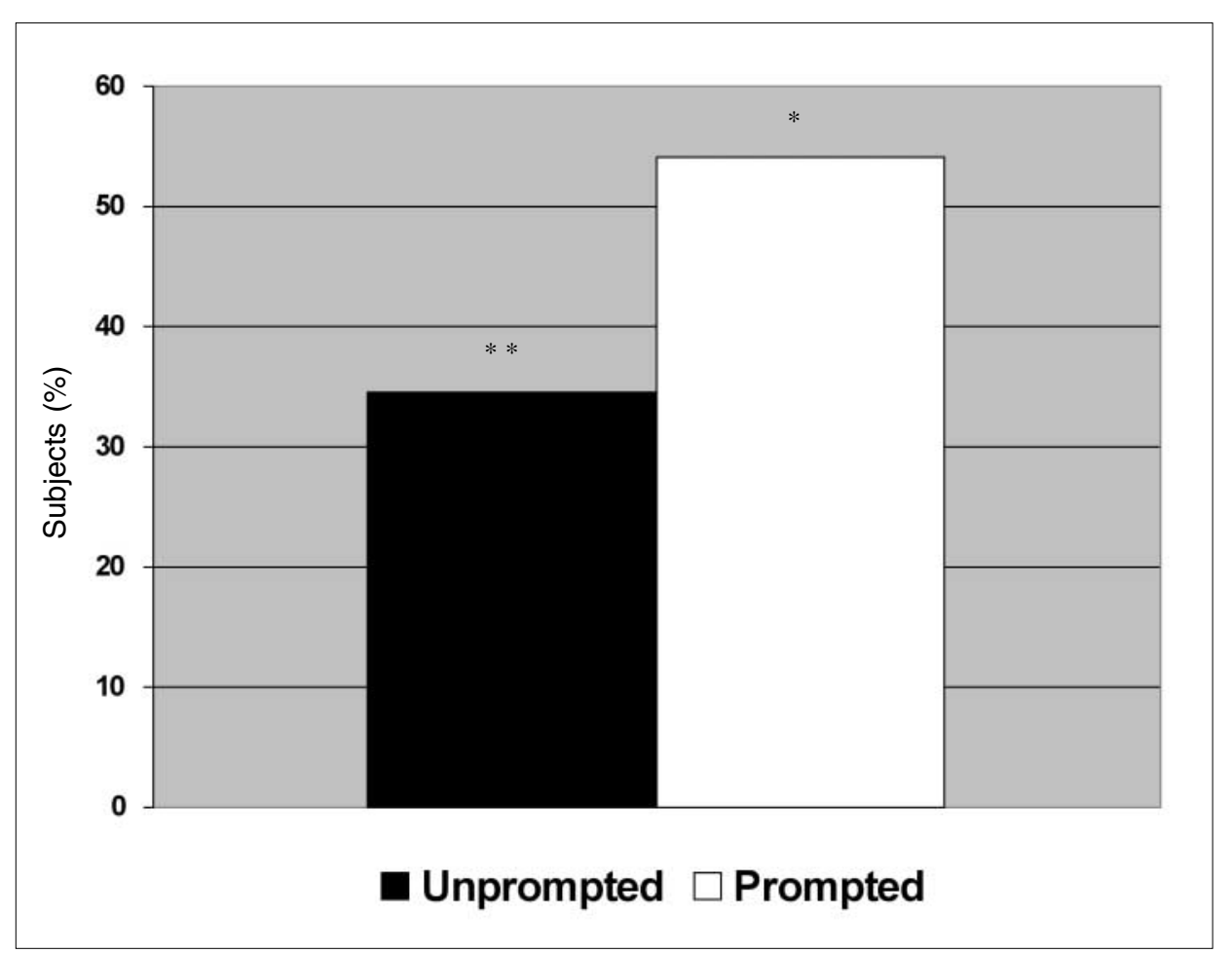


Table 3 Number and intensity of side effects assessed by the unprompted and prompted methods

\begin{tabular}{lrrr}
\hline & Unprompted & Prompted & $p$ \\
\hline $\begin{array}{l}\text { Number of different } \\
\text { adverse events, } \mathrm{n}(\%)\end{array}$ & & & \\
$\quad 2$ & $23(56)$ & $26(40.6)$ & 0.01 \\
2 & $14(34.1)$ & $22(34.3)$ & \\
$\quad 3$ or more & $4(9.7)$ & $16(25)$ & \\
Intensity of & & & \\
adverse events, $\%$ & 37.2 & 37.5 & NS \\
$\quad$ Mild & 39.2 & 42.5 & \\
$\quad$ Moderate & 23.6 & 20.0 & \\
$\quad$ Severe & &
\end{tabular}

Table 4 Common side effects in the unprompted and prompted questionnaires

\begin{tabular}{lrrrrr}
\hline Side effects & Unprompted & Prompted & $p$ \\
\hline Palpitation & $7(6.7 \%)$ & $18(17.3 \%)$ & $<0.001$ \\
Sleep disturbances & $8(7.6 \%)$ & $18(17.3 \%)$ & $<0.001$ \\
Sweating & $7(6.7 \%)$ & 18 & $(17.3 \%)$ & $<0.001$ \\
Difficulty of concentration & $4(3.8 \%)$ & 14 & $(13.4 \%)$ & $<0.0001$ \\
Weakness & $4(3.8 \%)$ & 4 & $(3.8 \%)$ & NS \\
Abdominal pain & $2(1.9 \%)$ & 3 & $(2.8 \%)$ & NS \\
Chest tightness & $2(1.9 \%)$ & 6 & $(5.7 \%)$ & $<0.01$ \\
Chest pain & $2(1.9 \%)$ & 9 & $(8.6 \%)$ & $<0.01$ \\
Tremor & $1(0.9 \%)$ & 5 & $(4.8 \%)$ & $<0.0001$ \\
\hline
\end{tabular}

Interestingly, two (1.9\%) subjects graded their adverse events as severe in the prompted questionnaire, but had not self-reported them. Most side effects were significantly more frequently reported in the prompted method. While only $1.9 \%$ of the subjects reported chest tightness and chest pain in the unprompted method, $5.7 \%$ and $8.6 \%$ of them $(p<0.01)$, respectively, reported these symptoms in the prompted method. Palpitations were self-reported by $6.7 \%$ of the subjects, and actively reported by $17.3 \%$ of them $(p<0.001)$; sleep disturbances were self-reported by $7.6 \%$ of the subjects, and actively reported by $17.3 \%$ $(p<0.001)$; sweating was self-reported by $6.7 \%$ of the subjects, and actively reported by $17.3 \%(p<0.001)$; difficulty concentrating was self-reported by $3.8 \%$ of the subjects, and actively reported by $13.4 \%(p<0.0001)$ (Table 4$)$.

\section{Discussion}

The triptans are generally very well tolerated, with less than half of patients reporting adverse events, mostly mild in intensity and transient [2, 10-12]. The methods used to assess tolerability often are not described in detail in randomized clinical trials on triptans. It is difficult to assess, for a particular trial, how information on side effects was collected and whether lists of side effects were presented. While some trials do not report the methods used to collect side effects [14], others used what seems to be an unprompted method [15]. There are methodological differences even when the unprompted method is used. In some trials, patients record the side effects in a headache diary [15], while in others the collection is also complemented by communication with the study coordinator [16]. Finally, some studies actively searched for the "incidence and nature of all serious adverse events (at any time before or after administration)" [17]. In our preliminary data, we showed that when assessing triptan adverse events, the method of data collection may dramatically influence the results. We also showed that from those subjects who did not self-report adverse events after using a triptan, most checked one or more of these events when presented with a list of possible adverse events. Even the rates of more dramatic side effects (like palpitations, chest pain, or chest tightness) may dramatically differ according to the methodology of data collection used. At first sight, it seems that prompted methods are more sensitive in assessing side effects, but on the other hand, it is important to note, as some authors argue, that patients given considerable information about the side effects of drugs substantially overestimate their personal risk. For example, the European Union guidelines recommend use of qualitative descriptions for five bands of risk, ranging from very rare (affecting $<0.01 \%$ of the population) to very common (>10\%) [17]. A study assessed 360 adults who were given either qualitative descriptions of drug side effects ("common" and "rare") or corresponding quantitative descriptions (2\% and $0.02 \%)$. Participants given the term "common" rated their personal risk of having a side effect as $50 \%$, whereas those given the equivalent numerical value of $2 \%$ rated their risk as $9.5 \%$; people who were told their chances of side effects were rare estimated their risk as $21 \%$, compared with $7 \%$ for patients who were given a quantitative risk of $0.02 \%$ [18]. Triptans are the drug of choice for most migraine sufferers who seek care $[1,2]$. Even though there are more similarities than differences among triptans, they do have important clinical differences. Our data support the following conclusions: (1) when assessing triptan (or any class of medication) adverse events, the method of data collection may dramatically influence the results; (2) some patients who do not self-report adverse events after using a triptan do in fact have side effects (44.3\%); (3) care should be taken when comparing the tolerability profile of triptans that were not in fact compared in head-to-head clinical trials with symmetrical design. 


\section{References}

1. Matchar DB, Young WB, Rosenerg J, et al (2000) Multispecialty consensus on diagnosis and treatment of headache: pharmacological management of acute attacks. Neurology 54:www.aan.com/public/practiceguidelines/03.pdf

2. Rapoport AM, Tepper SJ, Bigal ME, Sheftell FD (2003) The triptan formulations: how to match patients and products. CNS Drugs 17:431-447

3. Rapoport AM (2001) Frovatriptan: pharmacological differences and clinical results. Curr Med Res Opin 17 [Suppl 1]:s68-s70

4. Lipton RB, Hamelsky SW, Dayno JM (2002) What do patients with migraine want from acute migraine treatment? Headache 42[Suppl 1]:3-9

5. Ferrari MD, Roon KI, Lipton RB, Goadsby PJ (2001) Oral triptans (serotonin 5-HT(1B/1D) agonists) in acute migraine treatment: a meta-analysis of 53 trials. Lancet 358:1668-1675

6. Sheftel FD, Feleppa M, Tepper SJ et al (2004) Patterns of use of triptans and reasons for switching them in a tertiary care migraine population. Headache 44(7):661-668

7. Rapoport AM, Tepper SJ (2001) Triptans are all different. Arch Neurol 58:1479-1480
8. Headache Classification Committee of the International Headache Society (1988) Classification and diagnostic criteria for headache disorders cranial neuralgia and facial pain. Cephalalgia 8 [Suppl 7]:1-96

9. Welch KM, Mathew NT, Stone P, Rosamond W, Saiers J, Gutterman D (2000) Tolerability of sumatriptan: clinical trials and post-marketing experience. Cephalalgia 20:687-695

10. Nappi G, Sandrini G, Sances G (2003) Tolerability of the triptans: clinical implications. Drug Saf 26:93-107

11. Tepper SJ, Millson D (2003) Safety profile of the triptans. Expert Opin Drug Saf 2:123-32

12. Robbins L (2004) Frequent triptan use: observations on safety issues. Headache 44:178-182

13. Tfelt-Hansen P, Block G, Dahlof C, Diener HC, Ferrari MD, Goadsby PJ, Guidetti V, Jones B, Lipton RB, Massiou H, Meinert C, Sandrini G, Steiner T, Winter PB (2000) International Headache Society Clinical Trials Subcommittee. Guidelines for controlled trials of drugs in migraine: second edition. Cephalalgia 20:765-786
14. Goldstein J, Ryan R, Jiang K, Getson A, Norman B, Block GA, Lines C (1998) Crossover comparison of rizatriptan $5 \mathrm{mg}$ and $10 \mathrm{mg}$ versus sumatriptan $25 \mathrm{mg}$ and $50 \mathrm{mg}$ in migraine. Rizatriptan Protocol 046 Study Group. Headache 38:737-747

15. Eletriptan Steering Committee in Japan (2002) Efficacy and safety of eletriptan $20 \mathrm{mg}, 40 \mathrm{mg}$ and $80 \mathrm{mg}$ in Japanese migraineurs. Cephalalgia 22:416-423

16. Dowson AJ, Massiou H, Lainez JM, Cabarrocas X (2002) Almotriptan is an effective and well-tolerated treatment for migraine pain: results of a randomized, double-blind, placebo-controlled clinical trial. Cephalalgia 22:453-461

17. Dowson AJ, Charlesworth BR, Purdy A, Becker WJ, Boes-Hansen S, Farkkila M (2003) Tolerability and consistency of effect of zolmitriptan nasal spray in a long-term migraine treatment trial. CNS Drugs 17:839-851

18. Li Bassi L, Bertele V, Garattini S(2003) European regulatory policies on medicines and public health needs. Eur J Public Health 13:246-251

19. Berry DC, Knapp P, Raynor DK (2002) Provision of information about drug side-effects to patients. Lancet 359:853-854 